\title{
Commissioning and Initial LHC Run-2 Operation of the ATLAS Minimum Bias Trigger Scintillators
}

\author{
M. Hoffmann on behalf of the ATLAS Collaboration ${ }^{\text {a }}$ \\ ${ }^{a}$ DSM/IRFU (Institut de Recherches sur les Lois Fondamentales de l'Univers), CEA Saclay (Commissariat a l'Energie Atomique), Gif-sur-Yvette, France
}

\begin{abstract}
The Minimum Bias Trigger Scintillators (MBTS) are sub-detectors in ATLAS delivering the primary trigger for selecting events from low luminosity proton-proton, lead-lead and lead-proton collisions with the smallest possible bias. The MBTS have undergone a complete replacement before LHC Run-2 and several improvements have been implemented in the layout. Since 2014 the MBTS have been commissioned with cosmic rays and first LHC Run-2 beam splash events. We summarise the outcome of the commissioning.
\end{abstract}

Keywords: Scintillation detectors, detector characterisation and commissioning

\section{Introduction}

In the LHC data-taking period spanning years 2010-2013, known as LHC Run-1, the ATLAS ${ }^{1}$ detector [1] used a dedicated set of sub-detectors, the Minimum Bias Trigger Scintillators (MBTS), to trigger on events with minimal bias in protonproton and heavy-ion collisions. The radiation dose from LHC Run-1 ( $\approx 10^{4} \mathrm{~Gy}$ ) degraded the transparency of the scintillating medium in the MBTS by around 50\%, making them largely inoperable. Because of this, the MBTS underwent a complete replacement in preparation for the data-taking period foreseen in years 2015-2017, known as LHC Run-2.

\section{MBTS Layout for LHC Run-2}

The ATLAS MBTS are scintillation detectors composed of $2 \mathrm{~cm}$ thick doped polystyrene. They are mounted as disks around the LHC beam pipe on both A and C sides ${ }^{2}$ of ATLAS at a distance of $\pm 3.6 \mathrm{~m}$ from the interaction point [1]. Each disk consists of 8 wedges, which are composed of an inner and outer sector covering pseudorapidities $2.78<|\eta|<3.75$ and $2.08<|\eta|<2.78$, respectively. In both sectors, the emitted light from the scintillator is collected with 8 wavelength shifting optical fibers (WLS) that are embedded into grooves at the edges of the scintillator. These WLS are connected to clear optical fibers, which transport the light to photomultipliers that are read out by the ATLAS Tile Calorimeter electronics.

The layout of the MBTS designed for LHC Run-2 has undergone several improvements with respect to LHC Run-1. In order to maximise and homogenise the light yield, both sectors of each wedge have been merged from two pieces into one, and a more favourable WLS routing scheme has been opted for. In

\footnotetext{
${ }^{1}$ A Toroidal LHC ApparatuS

${ }^{2}$ The two sides of the interaction point
}

the early phases of LHC Run-2 operation, the outer sectors are read out with just 4 WLS. Once radiation damage has degraded the transparency of the scintillating medium, light yield can be recuperated by using all 8 WLS that are installed. In this way efficiency will be maintained as the detector ages.

\section{Commissioning with Cosmic Rays}

One fully assembled LHC Run-2 MBTS wedge was tested in a laboratory setup before integration with the ATLAS detector. The charge response induced by cosmic radiation was measured and the light yield from the inner and outer sector and the attenuation of the clear fibers used for light transport were extracted. An example of a MBTS charge spectrum measured with the inner sector read out with $2.1 \mathrm{~m}$ clear fiber is presented in Figure 1. The light yield was determined by fitting a function to the charge distributions [3]:

$$
f(x)=N \cdot \sum_{i=1}^{n}\left[\frac{e^{-\mu} \cdot \mu^{i}}{i !} \cdot \frac{1}{\sqrt{2 \pi} \sqrt{i \cdot \sigma_{1 \mathrm{PE}}^{2}+\sigma_{\mathrm{ped}}^{2}}} \cdot e^{\frac{-\left(x-\mu_{\mathrm{ped}}-i \cdot \mu_{\mathrm{PE}}\right)^{2}}{2 \cdot\left(i \cdot \sigma_{1 \mathrm{PE}}^{2}+\sigma_{\mathrm{ped}}^{2}\right)}}\right]
$$

which is a convolution of the Poisson distributions with a Gaussian. This reflects that the number of emitted photoelectrons follows a Poisson distribution and the photomultiplier response to $i$ photoelectrons has a Gaussian shape. Here the parameter $\mu$ is a direct measure of the number of photoelectrons. The remaining parameters are characteristics of the photomultiplier that were determined from an independent calibration.

The fit results for all the performed measurements are summarised in Figure 2. From this study, the following observations have been made; the light yield in the outer sector is $\approx 50 \%$ larger than in the inner sector when opting for all 8 available WLS in the readout. Furthermore, when only opting for the intended 4 WLS in the outer sector its yield is $\approx 30 \%$ smaller than in the inner sector. Moreover it is observed that 
the yield is doubled in the outer sector when going from 4 to 8 WLS. All these observations are in agreement with the expectation from the detector layout. Another finding is that the single-clad clear fibers intended for light transportation in LHC Run-1 had an unacceptably high attenuation length. For this reason, a double-clad fiber model was procured and installed in preparation for LHC Run-2.

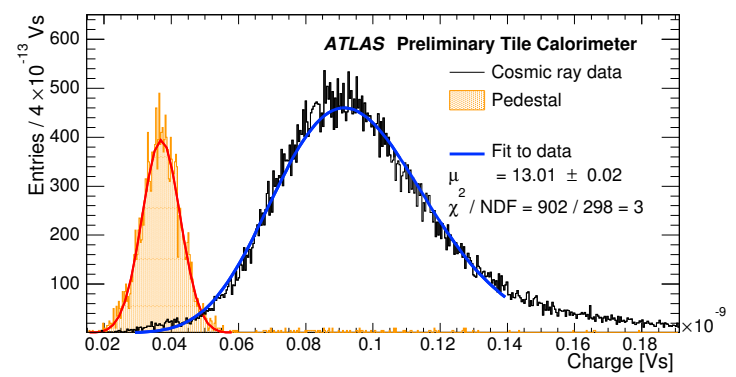

Figure 1: Distribution of integrated charge induced by cosmic radiation measured with the inner sector of a LHC Run-2 MBTS counter read out with $2.1 \mathrm{~m}$ clear fiber [2]. The fit to data (blue) based on Eq. (1) gives an estimate on the average number of photoelectrons. The orange distribution is the pedestal used for calibrating the photomultiplier.

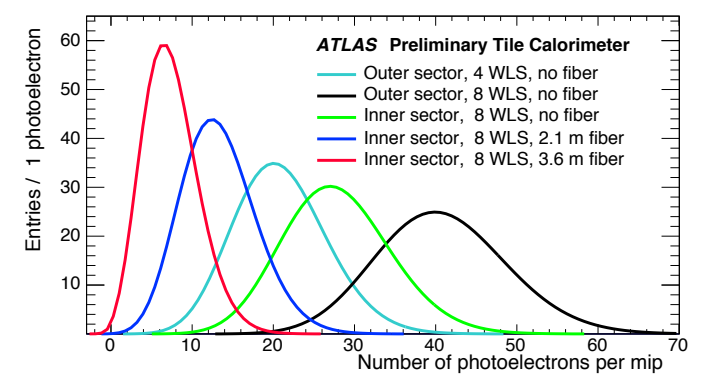

Figure 2: Summary of light yield measurements performed with a LHC Run-2 MBTS counter with cosmic radiation [2]. Each curve represents the fit to the integrated charge spectrum (with Eq. (1)) recorded with the inner and outer sector of the MBTS read out with 4 or 8 wavelength-shifting fibers read out with different clear fiber lengths. The normalisation is arbitrary.

\section{Initial LHC Run-2 Operation}

The LHC Run-2 MBTS counters were integrated into the ATLAS data acquisition chain in the ATLAS re-commissioning phase before LHC Run-2. The MBTS signals after shaping and amplification are passed to discriminators that send a NIM trigger signal to the ATLAS Central Trigger Processor [4]. In LHC Run-1 leading edge discriminators were deployed for this purpose. However, due to the large dynamic range of the photomultipliers, the signals were subject to a time walk. To prevent this, constant fraction discriminators were deployed instead for LHC Run-2.

The timing of the MBTS trigger signals was determined by grazing single proton beams against collimators, so-called beam splash events. For the measurement of the timing, triggering on the MBTS discriminator signal was done on the leading edge and the timing was determined with respect to an independent, single-sided calorimeter trigger on outgoing splash particles passing the interaction point. The results obtained with
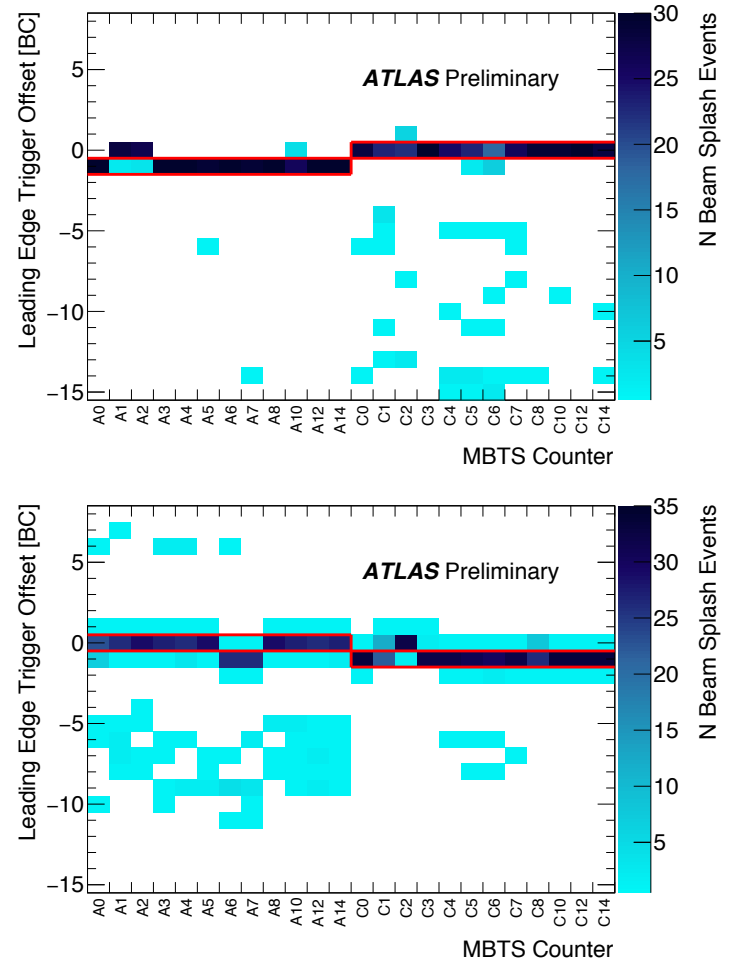

Figure 3: The timing of the MBTS counters determined with respect to a singlesided calorimeter trigger [5]. Data were taken with 35 beam splash events impinging from ATLAS side A (top) and 30 beam splash events impinging from ATLAS side C (bottom). The timing is presented in units of $25 \mathrm{~ns}$, which correspond to one bunch crossing. The in-time bunch crossing is highlighted with a red border. Counters 0-7 compose the ring of inner sectors, while counters 8 , 10,12 and 14 form the ring of outer sectors.

splash events impinging from either side A and side C in ATLAS are presented in Figure 3. As observed, the majority of channels are well-timed and within the in-time bunch crossing.

\section{Conclusion}

The commissioning of the LHC Run-2 MBTS with cosmic radiation indicates that the light yield of the detector is plentiful and in good agreement with the expectation from the detector layout. Moreover, the timing of the MBTS trigger signals determined from the first LHC Run-2 beam splash events shows that they are well-timed. In summary, measurements in the commissioning phase indicate that the MBTS are well-functioning and ready for the LHC Run-2 data taking.

\section{References}

[1] The ATLAS Collaboration, The ATLAS Experiment at the CERN Large Hadron Collider, JINST 3 (2008) S08003

[2] The ATLAS Collaboration, Approved Tile Calorimeter Plots https://twiki.cern.ch/twiki/bin/view/AtlasPublic/ApprovedPlotsTile

[3] S. Ask et al., Luminosity measurement at ATLAS - development, construction and test of scintillating fibre prototype detectors, Nucl. Instrum. Meth. A568 (2006) 588-600.

[4] A. Sidoti on behalf of the ATLAS TDAQ Collaboration, Minimum Bias Trigger Scintillators in ATLAS Run II, JINST 9 (2014) C10020.

[5] The ATLAS Collaboration, Minimum Bias Trigger Public Results https://twiki.cern.ch/twiki/bin/view/AtlasPublic/MinBiasTriggerPublicResults 